\title{
Diagnóstico prenatal y cribado de cromosomopatías
}

\author{
Ma N. Parga Soler, S. Martínez Machuca*, \\ O. Martín IDOETA, M. SÁnChez-PAStor RUIZ** \\ Médico de Familia. C.S. V Centenario. Área 5. Madrid. * M édico de Familia \\ C.S. Soto del Real. Área 5. Madrid. **Especialista en Obstetricia y Ginecología. \\ Servicio de Ginecología. Hospital La Paz. Madrid
}

\section{RESUMEN}

Actualmente, la puesta en marcha de programas de atención a la mujer embarazada ha permitido la realización sistemática de pruebas de screening que hacen posible conocer antes del parto el esta do de salud del feto. A lo largo de los últimos 15 años se han realizado relevantes progresos diag nósticos en medicina materno-fetal, y frecuente mente encontramos nuevas aportaciones que son motivo de controversia a la hora de incluirlos en programas de salud, valorando su aplicabilidad y coste-efectividad o eficiencia.

La facilidad de acceso a información científica es, en ocasiones, fuente de confusión para el profe sional y el paciente, por lo que a través de este ar tículo intentamos sintetizar la evidencia científica en cuanto a coste-efectividad y aplicabilidad de las estrategias actualmente asequibles de detección prenatal precoz, para que el profesional pueda contar con argumentos coherentes que ofrecer des de su consulta de Atención Primaria.

Palabras clave: Diagnóstico prenatal. Análisis coste-beneficio. Screening neonatal.
Prenatal diagnosis and chromosomal abnorma lities screening

\begin{abstract}
Currently, the setting up of pregnancy health programs have enabled systematic screeening tests that recognize the fetus' health prepartum. Over the last 15 years a significant diagnostic progress has been made in materno-fetal medicine, and we frequently find new contributions that are subject of controversy when included in health programs, regarding factors such as applicability and cost-ef fectiveness or efficiency.

The easy access to the scientific information can create in some ocassions confusion for the patient and the doctor. This article tries to sinthetize the cost-effectiveness scientific evidence and the strategic applications that are actually approachable for prenatal detection and to make it easier for the professional to be supported with coherent argu ments for the patient in his Primary Health Care center.
\end{abstract}

Key words: Prenatal diagnosis. Cost-benefit analysis. Neonatal screening.

\section{INTRODUCCIÓN}

Entendemos por screening de población para detección de anomalías o enfermedades, la aplicación sistemática de métodos que permitan seleccionar entre todos los individuos aparentemente sanos, aquéllos con mas riesgo de padecerlas. El diagnóstico prenatal engloba a todas aquellas actividades diagnósticas que buscan conocer la existencia de un defecto congénito, que, según la definición dada por la OMS incluye a toda anomalía del desarrollo morfológico, estructural, funcional o molecular, presente al nacer, aunque pueda manifestarse más tarde, externa o interna, familiar o esporádica, heredada o no, única o múltiple.

La trisomía 21 o síndrome de Down (SD) ha sido el objetivo prioritario en el diagnóstico de anomalías cromosómicas fetales, ya que es la aneu- 
ploidía más frecuente en nacidos, causa común de retraso mental severo y supervivencia postnatal más prolongada, no existiendo ningún método de prevención primaria.

Debemos tener en cuenta la tendencia actual de creciente aumento de gestantes en la franja de edad de 35 y 38 años, así como la disponibilidad de recursos materiales y humanos en el área de actuación que condicionan la aplicación sistemática de una determinada estrategia de cribado en gestantes mas jóvenes ${ }^{1-3}$.

\section{INFECCIONES DE TRANSMISIÓN PERINATAL. DIABETES GESTACIONAL}

Las pruebas recomendadas en nuestro medio para el seguimiento del embarazo en todas las gestantes, independientemente de su edad o la presencia o no de factores de riesgo incluyen las de infecciones de transmisión perinatal: rubeola, sífilis, hepatitis B, toxoplasmosis, VIH, y más recientemente Es treptococo Agalactiae; la realización del test de O’Sullivan a las 24 semanas y la realización de tres exploraciones ecográficas, realizadas la primera entre la 8-12 semanas, la segunda entre la 18-20 semanas y la tercera entre la 34-36 semanas.

A continuación se recoge una relación de las características principales de dichas pruebas y se incluye la referencia para la pertinencia o no de su realización, según los criterios de la U.S. Preventi ve Services Task Force, que clasifica el grado de fuerza de la recomendación según el grado de evidencia científica, en cinco niveles ${ }^{2,4}$ (Tabla I).

\section{Tabla I}

FUERZA DE LA RECOMENDACIÓN SEGÚN GRADO DE EVIDENCIA CIENTÍFICA

Existen evidencias sólidas para apoyar la recomendación de considerar específicamente el trastorno en un examen de salud periódico.

Hay pocas evidencias para apoyar la recomendación de considerar específicamente el trastorno en un examen de salud periódico.

No existen evidencias suficientes para recomendar 0 rechazar la inclusión del trastorno en un examen de salud periódico, pero es posible hacer recomendaciones apoyándose en otros criterios.

Hay pocas evidencias para apoyar la recomendación de no considerar el trastorno en un examen de salud periódico.

Existen evidencias sólidas para apoyar la recomendación de no considerar el trastorno en un examen de salud periódico ${ }^{2,4}$.

\section{DIABETES GESTACIONAL}

\section{Test de O'Sullivan}

Como es conocido se trata de un test diagnóstico de diabetes gestacional, su positividad es factor de riesgo para desarrollo posterior de diabetes y macrosomia fetal.

La técnica consiste en administración 50 gramos de glucosa oral y la determinación de la glucemia una hora después. El test es positivo si la glucemia es mayor de $140 \mathrm{mg} / \mathrm{dl}$ o 7,8 mmol/L. El test de confirmación se hace con sobrecarga de 100 g glucosa. Su objetivo es el tratamiento precoz de la diabetes gestacional para prevenir la macrosomía fetal, así como la disminución de la morbi-mortalidad materna.

Presenta una sensibilidad del $71-83 \%$ y una especificidad de $78-87 \%{ }^{4}$. La indicación de la semFYC es la realización del test a toda gestante mayor de 25 años y menores de esta edad con factores de riesgo. Debido a la existencia de varios métodos para el diagnóstico de diabetes gestacional y la controversia sobre la idoneidad del más adecuado y la evidencia de que sólo un 5\% de macrosomías fetales son debidas a diabetes gestacional, la recomendación de la realización sistemática de esta prueba está actualmente en discusión. U.S. Preventive Services Task Force, nivel de recomen dación " $C$ " 4,5

\section{ENFERMEDADES TRANSMISIBLES}

\section{Toxoplasmosis}

Consiste en la detección de antígenos parasitarios circulantes en fluidos corporales mediante la técnica ELISA, específico pero de sensibilidad variable. La interpretación de resultados se expone a continuación (Tabla II). Su objetivo es la prevención del contagio e infección materna para evitar la infección y afectación fetal. El tratamiento con espiramicina hasta finalizar el embarazo reduce la transmisión fetal en un $60 \%$.

La indicación actual en nuestro medio es la determinación en todas las gestantes al inicio de la gestación, repitiéndose la determinación en gestantes no inmunes, no estando bien establecida la periodicidad de la misma ${ }^{7}$ (Tabla II).

\section{Virus de hepatitis B}

Detección en sangre de antígeno de superficie de virus de hepatitis B: Hbs Ag mediante ELISA. Presenta una sensibilidad y especificidad superior al $98 \%{ }^{8}$. Su objetivo es la prevención de la enfermedad en el recién nacido. La administración de la va- 
INTERPRETACIÓN DE LA RESPUESTA SEROLÓGICA A LA TOXOPLASMOSIS

SEGÚN EL MOMENTO DE REALIZACIÓN DE LA PRUEBA

\begin{tabular}{|c|c|c|}
\hline GESTACIÓN & $\lg G$ & INTERPRETACIÓN \\
\hline No embarazo & $\begin{array}{l}\text { Negativa } \\
\text { Positiva }\end{array}$ & $\begin{array}{l}\text { Control primer mes de gestación } \\
\text { Inmune }\end{array}$ \\
\hline Primer mes & $\begin{array}{l}\text { Negativa } \\
\text { Positiva }\end{array}$ & $\begin{array}{l}\text { No inmune. Susceptible. Seguimiento. } \\
\text { Posibilidad de infección periconcepcional. } \\
\text { No otros tipos de pruebas. Riesgo mínimo. No } \\
\text { tratamiento médico. }\end{array}$ \\
\hline Segundo mes & $\begin{array}{l}\text { Negativa } \\
\text { Positiva }\end{array}$ & $\begin{array}{l}\text { No inmune. Susceptible. Seguimiento. } \\
\text { Posibilidad de infección periconcepcional. No otros } \\
\text { tipos de pruebas. Riesgo mínimo. No tratamiento } \\
\text { médico. }\end{array}$ \\
\hline Tercer mes & $\begin{array}{l}\text { Negativa } \\
\text { Positiva }\end{array}$ & $\begin{array}{l}\text { No inmune. Susceptible. Seguimiento. } \\
\text { Posibilidad de infección peri o concepcional. Estudio } \\
\text { segunda muestra: } \\
\text { IgG estable: no en periodo evolutivo. Inmune. No } \\
\text { tratamiento. } \\
\text { IgG aumento: posible infección reciente, } \\
\text { reactivación, reinfección. Estudio de IgM, IgA, } \\
\text { avidez, IgG. } \\
\text { Tratamiento. Ecografías. Funiculocentesis }\end{array}$ \\
\hline Cuarto mes & $\begin{array}{l}\text { Negativa } \\
\text { Positiva }\end{array}$ & $\begin{array}{l}\text { No inmune. Susceptible. Seguimiento. } \\
\text { Títulos bajos, medios: segunda muestra } \\
\text { IgG estable: inmune. } \\
\text { IgG en aumento: apartado tercer mes. } \\
\text { Títulos altos: IgM: } \\
\text { Negativa: inmune. No tratamiento } \\
\text { Positiva: IgA, avidez-IgG. Posible infección } \\
\text { reciente. Tratamiento. Ecografías. } \\
\text { Funiculocentesis. }\end{array}$ \\
\hline $\begin{array}{l}\text { Controles (en caso de } \\
\lg (-) \text { en los anteriores) }\end{array}$ & $\begin{array}{l}\text { Negativa } \\
\text { Positiva }\end{array}$ & $\begin{array}{l}\text { No inmune. Susceptible. Seguimiento. } \\
\text { Infección reciente. IgM, IgA, avidez-IgG. Ecografías. } \\
\text { Funiculocentesis. IVE o tratamiento. }\end{array}$ \\
\hline
\end{tabular}

cuna para la hepatitis B combinada con una dosis única de inmunoglobulina específica para hepatitis $\mathrm{B}$ administrada en las 12 horas desde el nacimiento tiene una eficacia del $75-95 \%$ para prevenir la infección crónica en lactantes nacidos de madres portadoras de $\mathrm{HBs} \mathrm{Ag}^{4,8}$.

Indicaciones: determinación a todas las gestantes al inicio de la gestación. La prueba puede repetirse en el tercer trimestre si se sospecha hepatitis aguda, exposición a hepatitis o la mujer presenta conducta de riesgo elevado. U.S. Preventive Servi ces Task Force, nivel de recomendación " $A$ " 4,9 .

\section{Sífilis}

Realización de pruebas no treponémicas (VDRL o RPR). La especificidad es del 75-85\% en personas con enfermedades o trastornos preexistentes y se acerca al $100 \%$ en personas sanas ${ }^{10}$. La sensibilidad es del $62-76 \%{ }^{4,10}$. Debido a la presencia de falsos positivos, todos los resultados reactivos positivos deben confirmarse con pruebas treponémicas.

Con la administración precoz de penicilina en el embarazo prevenimos la sífilis congénita, muerte fetal o perinatal $(40 \%$ de los embarazos afecta- 
dos) $)^{4,6}$ así como morbilidad neonatal. La determinación serológica está indicada en todas las gestantes en la primera visita. En mujeres de riesgo se recomienda repetir la determinación en el tercer trimestre y después del parto. U.S. Preventive Services Task Force, nivel de recomendación " $A$ ".

\section{VIH}

Se realiza el cribado inicial con enzimoinmunoanálisis (EIA). Los resultados se consideran reactivos cuando se ha confirmado el resultado positivo en una segunda prueba de la muestra original. La sensibilidad en personas con SIDA clínico es casi del $100 \%$, pero en las personas recién infectadas pueden no detectarse anticuerpos hasta semanas o meses después. La especificidad es superior al $99,5 \%$ en casi todas las pruebas ${ }^{11}$. Para la confirmación se utiliza la prueba de Western Blot, con una especificidad del $100 \%$.

Objetivo: diagnóstico precoz para reducir la transmisión perinatal del virus. La utilización de fármacos antirretrovirales entre la semana 14 y 34 de gestación, prolongada durante el parto y durante 6 semanas en recién nacidos, reduce significativamente la infección perinatal por VIH. La realización de una cesárea también se acompaña de tasas más bajas de transmisión vertical. Otras ventajas serían el inicio de quimioprofilaxis de enfermedades asociadas al VIH si fuera preciso, realización de pruebas en los compañeros sexuales y consejo sobre el uso del preservativo y la actuación precoz de los servicios sociales para facilitar el cuidado de la madre y el recién nacido.

Indicacion: en España se aplica a todas las gestantes en la primera visita. Se debe repetir la determinación en gestantes con conductas de riesgo. Para la U.S. Preventive Services Task Force el cribado en mujeres de riesgo o en comunidades con prevalencia de recién nacidos seropositivos mayor al $0,1 \%$ tiene un nivel de recomendación " $\mathrm{A}$ " y en comunidades de baja prevalencia un nivel de recomendación " $\mathrm{C}$ " 4,12.

\section{Rubeola}

Detección de anticuerpo de inhibición de la hemaglutinación (HI), enzimoinmunoanálisis (EIA) y aglutinación con látex. Presenta una sensibilidad del $92-100 \%$ y especificidad del $71-100 \%{ }^{13}$. El objetivo es la detección de mujeres no inmunes para realizar vacunación tras el parto y prevenir posibles infecciones fetales en un segundo embarazo. Los esfuerzos destinados a prevenir la rubeola congénita se deben centrar en la vacunación universal en la niñez $y$, debido a que se estima que entre un 6-12\% de la población adulta joven es seronegativa, en la vacunación a jóvenes y adultos jóvenes susceptibles, en particular a mujeres en edad fértil ${ }^{4,6,13}$.

\section{Estreptococo betahemolítico del grupo B}

La técnica consiste en el cultivo en medio selectivo. Se obtiene una muestra procedente de tercio inferior vaginal y anorrectal, cuanto más próxima al parto se tome la muestra mayor es el grado de correlación con el estado de portadora. Su objetivo es la prevención de la infección neonatal.

La indicación no está bien establecida actualmente. Las opciones van desde el cribado universal a todas las gestantes al tratamiento intraparto de mujeres con factores de riesgo sin conocer su estado de portadoras. El protocolo más utilizado es el que se expone, según la edad gestacional ${ }^{14-16}$ :

36 semana: cultivo sistemático:

Resultado + : tratamiento intraparto

Resultado - : no tratamiento

Menor de la semana 36: tratamiento intraparto siempre si no dispone de cultivo

Mayor de la semana 37 sin cultivo: tratamiento si factores de riesgo

\section{CRIBADO DE CROMOSOMOPATÍAS}

La prevalencia global de anomalías cromosómicas en nacidos es el 0,6, 4-5\% de concepciones ${ }^{1}$, que en la mayoría son letales en las primeras semanas de gestación. Las cifras globales en España muestran un descenso en la prevalencia de SD en nacidos vivos del 1,49/1.000 en el año 1976 al 1,09/1.000 en el 1988, que es de suponer se deba al impacto de la introducción del diagnóstico prenatal y la interrupción voluntaria de embarazo (IVE). Aproximadamente el 54\% de las anomalías cromosómicas se presentaron en gestantes mayores de 35 años. Del total de gestantes con diagnóstico de cromosomopatías, el $56 \%$ optaron por IVE ${ }^{1}$.

En los años 70 se introdujo progresivamente la aplicación de amniocentesis en el segundo trimestre como prueba diagnóstica de aneuploidías, particularmente para el SD, en gestantes con riesgo relacionado a la edad materna. Paralelamente la determinación de alfa-fetoproteína (AFP) en suero materno marcó el inicio en la utilización de "marcadores" bioquímicos en el cribado de aneuploidía fetal en el segundo trimestre de gestación. Posteriormente se han descubierto otros marcadores bioquímicos o ecográficos. El riesgo global estimado de interrupción de embarazo tras amniocentesis o biopsia corial, para todas las edades, es del 0,5-1,5\% $\%^{1,6}$.

La práctica actualmente más extendida es la aplicación de los métodos de cribado en el segundo 
trimestre, y es práctica comúnmente aceptada en el control de gestación de bajo riesgo; aunque hablaremos de estrategias más recientes pero actualmente asequibles, para su aplicación en el primer trimestre con las consiguientes ventajas relacionadas con su precocidad ${ }^{1,6,17,18}$.

\section{¿A QUÉ GESTANTES VA DIRIGIDO EL DIAGNÓSTICO PRENATAL DE CROMOSOMOPATÍAS?}

Las gestantes con bajo riesgo de ser portadoras de un feto afecto de cromosomopatía, detectable mediante el método de cribado son el objetivo prioritario, ya que la edad materna las sitúa en el sector de bajo riesgo. Ello no excluye que aquellas embarazadas de alto riesgo que por razones personales deseen disponer de más información para decidir la práctica de una técnica invasiva, puedan acogerse al cribado.

Los factores de riesgo para presentar un feto afecto de cromosomopatía son:

$\longrightarrow 35$ años.

-Antecedentes de embarazo previo con anomalía cromosómica.

- Antecedentes de SD en la familia, nacidos de embarazos entre los 21 y los 35 años.

-Progenitor portador de anomalía cromosómica.

- Abortos de repetición, nacidos muertos o malformaciones congénitas o esterilidad sin causas establecidas $^{1,19}$.

La selección para recomendar las técnicas invasivas de diagnóstico se puede realizar por tres fuentes de información:

—Datos clínicos y epidemiológicos.

- Cribado bioquímico en suero materno.

-Datos obtenidos de la exploración ecográfica.

Los criterios epidemiológicos basados en la edad, antecedentes reproductivos y familiares han mostrado eficacia muy escasa. La edad como criterio aislado genera un número considerable de falsos negativos (FN), todos los SD generados en $<35$ años, que son aproximadamente el $60 \%$.

Debe establecerse un límite de riesgo arbitrario a partir de los resultados de cribado (edad materna, marcadores bioquímicos y ecográficos) para ofrecer una prueba diagnóstica, ya que las variables de cribado son cuantitativas continuas.

El impacto que la aplicación de estas estrategias de cribado ha tenido sobre la prevalencia para el SD se ha experimentado básicamente en las gestantes mayores de 34 años, dada la escasa o nula implementación clínica de los criterios de cribado en el grupo de mujeres menores de 35 años. Por todo esto las gestantes de "bajo riesgo" serían el objetivo prioritario del cribado ${ }^{1,20}$.
El punto de corte en 35 años para la realización de tests diagnósticos (amniocentesis o biopsia corial) ha sido elegido básicamente por 4 razones:

1. El deseo de restringir el acceso a los servicios de diagnóstico prenatal por la limitada disponibilidad de los mismos.

2. El análisis coste-beneficio es favorable a partir de esta edad; los costes derivados de ofrecer test serían más que compensados por el ahorro asociado a la prevención de nacimientos de niños con SD

3. La incidencia de SD muestra un incremento de riesgo notable a partir de esta edad. Esto es así cuando las prevalencias se calculaban para intervalos de 5 años y la representación gráfica daba un salto cuantitativo a los 35 años. Si se representan a intervalos de 1 año, el aumento de la prevalencia es gradual, aunque la idea de los 35 años persiste como base para indicar un estudio citogenético.

4. Los análisis riesgo-beneficio sugieren que el riesgo de la enfermedad sería mayor que el derivado del procedimiento diagnóstico. A esta edad el riesgo de aborto tras amniocentesis o biopsia corial es similar al de tener un feto afectado de $\mathrm{SD}^{21}$.

\section{¿QUÉ MARCADORES BIOQUÍMICOS Y CUÁNDO REALIZAR EL CRIBADO?}

\section{$2^{\circ}$ Trimestre}

El denominado triple screening surge de la observación, en 1984, de la relación entre la presencia de SD y descenso de niveles séricos maternos AFP. Posteriormente se añade el incremento hCG y el descenso del estriol no conjugado uE3. Combinando el triple screening con la edad materna obtenemos un índice de detección para SD del 60-69\%, con un $5 \%$ de falsos positivos (FP), en gestantes menores de 35 años, y para un punto de corte 1:250 $0^{1,22}$.

Si prescindimos del uE3 obtenemos índices de detección similares con descenso de FP; y es el modelo más ampliamente utilizado actualmente (edad materna + AFP + hCG), no existiendo consenso actual para asociar estriol no conjugado o inhibina A.

El periodo óptimo de realización está comprendido entre la $15^{\mathrm{a}}$ y $16^{\mathrm{a}}$ semana de gestación, pudiéndose hacer hasta la semana 20 sin pérdida de eficacia, aunque debemos considerar el tiempo de amniocentesis o BC y el límite legal de IVE (semana 22$)^{1,18,22}$.

\section{$1^{0}$ Trimestre}

Existe acuerdo en la utilización de hCG (preferentemente la fracción libre, $\mathrm{f} \beta$-hCG) y la proteí- 
na específica de la placenta presente en plasma materno (PAPP-A), esta última con mayor capacidad discriminatoria cuanto menor edad gestacional.

El descenso en PAPP-A combinado con el aumento de $\mathrm{f} \beta$-hCG presenta una sensibilidad del $65 \%$ para un porcentaje de FP del $5 \%{ }^{1,22}$. Debe medirse antes de la semana $12^{1}$.

\section{Marcadores ecográficos}

No existen revisiones sistemáticas ni ensayos randomizados controlados en torno a la ecografía del $1^{\circ}$ trimestre, por lo que no hay suficiente evidencia para utilizarla como screening. La ecografía antes de la semana 24 (normalmente entre la semana 18-20) permite la detección de anomalías cromosómicas asociadas a determinadas malformaciones estructurales.

El pliegue o traslucencia nucal (no todos los autores admiten que se trate de la misma entidad) fue descrito como marcador de aneuploidía fetal en el $2^{\circ}$ trimestre con una sensibilidad del $40 \%$ para un porcentaje de FP $<1 \%{ }^{1}$, siendo la sensibilidad para el SD del 62 al $73 \%$ para un $4 \%$ de $\mathrm{FP}^{1,23}$. Otros autores concluyen diciendo que los análisis coste-beneficio para la ecografía del $2^{\circ}$ trimestre, depende de su exactitud diagnóstica, y sólo sería beneficiosa de rutina cuando su sensibilidad global para el SD fuera mayor de $74 \%$, o cuando ésta fuera realizada únicamente en centros terciarios, ya que su sensibilidad depende fundamentalmente de la edad materna y de la experiencia del explorador ${ }^{1,18,20,24-26}$.

\section{COMBINACIÓN DE MARCADORES BIOQUÍMICOS Y ECOGRÁFICOS}

\section{$2^{\circ}$ Trimestre (semana 15-18)}

Actualmente es la mejor alternativa, aunque se aplica en pocos centros. La combinación de criterios bioquímicos (AFP, hCG) y ecógraficos (pliegue nucal) mostró una sensibilidad del $78 \%$ para un porcentaje de FP del $14 \%^{1,27}$.

\section{$1^{0}$ Trimestre (semana 10-13)}

La sensibilidad oscila alrededor del $86-90 \%$ para un $5 \%$ de $\mathrm{FP}^{22}$, resultados muy superiores a los obtenidos en el $2^{\circ}$ trimestre, pero no existe consenso para apoyar la combinación de marcadores bioquímicos y ecográficos en el $1^{\circ}$ trimestre en poblaciones de bajo riesgo. El cribado del $1^{\circ}$ trimestre sería razonable cuando se pueda garantizar la calidad de la exploración ecográfica, por lo que puede considerarse como una alternativa de futuro ${ }^{1,22,23}$.

\section{Test integrado}

Algunos autores proponen el test integrado que consiste en la combinación de marcadores de $1^{\circ} \mathrm{y}$ $2^{\circ}$ trimestre:

$1^{\circ}$ trimestre: PAPP-A más traslucencia nucal.

$2^{\circ}$ trimestre: AFP, hCG, inhibina A y uE3.

Obtienen una sensibilidad del 94 ó 85\%, dependiendo del índice de FP, un 5 y un $0,9 \%$ respectivamente, con lo que la necesidad de amniocentesis o $\mathrm{BC}$ se reduce considerablemente. Concluyen que el test integrado hace el screening y el diagnóstico prenatal mucho más seguros y efectivos que los métodos actualmente disponibles.

La principal desventaja es que requiere una visita precoz de la gestante a su médico de Atención Primaria o al tocólogo, entre la semana 10-13, requiriendo una nueva extracción a las 5 semanas aproximadamente ${ }^{22}$.

\section{METODOLOGÍA PARA EL CRIBADO DE ANEUPLOIDÍAS}

\section{Estimación de riesgo}

La estimación del riesgo, a priori, para la edad de la paciente, se obtiene del meta-análisis de Cuckle, que expresa dicho riesgo en el momento del parto. La dificultad principal está en ajustar el tiempo exacto de gestación por ecografía y además la concentración de los distintos marcadores en suero materno varía con el tiempo de gestación, por lo que el valor del marcador debe ser transformado a múltiplos de la mediana (MoM) corregido según peso, raza, tabaquismo, DMID, número de fetos.

La estimación del riesgo puede efectuarse por diferentes métodos. El más utilizado es el like-hood o de probabilidad. La razón de probabilidad para un determinado marcador bioquímico o ecográfico se calcula según la distribución poblacional gaussiana para un grupo afecto y otro no afecto de la trisomía a detectar. Finalmente la estimación de riesgo se obtiene multiplicando la probabilidad $a$ priori para la edad de la paciente de ser portadora de dicha trisomía por la razón de probabilidad obtenida a partir de los marcadores, previamente transformados en MoM, y se expresa como un índice de probabilidad de 1 entre el resultado de dicho producto. Todo el proceso de cálculo se efectúa mediante un programa informático específico diseñado para este fin ${ }^{12,28}$.

El nivel de corte, es decir, el "riesgo" a partir del cual se ofrecerá un procedimiento invasivo de diagnóstico, es una decisión arbitraria y depende fundamentalmente de los recursos materiales que se puedan invertir en el programa en concreto. En 
el momento actual existe amplio consenso en España para utilizar en el segundo trimestre y para el SD un nivel que oscile entre 1:270 y 1:250, expresado en el momento del cribaje, que corresponde al riesgo de una mujer de 35 años de ser portadora de un feto afecto de SD. Para la toma de decisiones se utilizan comúnmente las curvas ROC (Receiver Operating Characteristic) en las que gráficamente se confrontan sensibilidad y especificidad para cada punto de corte arbitrariamente establecido. Combinando los marcadores, podemos establecer con mayor precisión el riesgo de aneuploidía fetal y evitaremos la práctica innecesaria de buen número de procedimientos invasivos (amniocentesis o biopsia corial) para el diagnóstico inequívoco ${ }^{1}$.

\section{GESTACIÓNMÚLTIPLE}

Las gestaciones gemelares presentan dificultades interpretativas si existe discordancia cromosómica gemelar. El valor de los marcadores en suero materno se estima al doble aproximadamente que en gestaciones únicas. En gestaciones de mayor orden el cribado bioquímico no es aplicable.

En cuanto a marcadores ecográficos, la traslucencia nucal presenta una sensibilidad del $50 \%$ en gestaciones dobles con un feto afecto de $\mathrm{SD}^{1}$.

\section{TRISOMÍAS 18 Y 13}

La amniocentesis no está justificada desde el punto de vista coste-beneficio ya que son poco prevalentes y las consecuencias de estas anomalías para la salud y costes son limitados.

Trisomía 18: los marcadores bioquímicos (triple screening) ofrecen una sensibilidad del $60 \%$ para un porcentaje de FP de $1 \%{ }^{1,29,30}$. Los marcadores ecográficos tienen una sensibilidad del $85 \%$ para un $4 \%$ de $\mathrm{FP}^{29}$.

\section{DEFECTOS DEL TUBO NEURAL}

Debe obtenerse una muestra de sangre a partir de la semana 15 , donde encontraremos en los defectos de tubo neural la AFP aumentada con una sensibilidad del $75 \%$ para una tasa de FP del 3\%, para un punto de corte $2,5 \mathrm{MoM}^{1,30}$.

\section{CONCLUSIÓN}

Dada la baja prevalencia de cromosomopatías, el valor predictivo positivo y el valor predictivo negativo poseen escaso valor como indicador de efectividad. Los mejores indicadores son sensibilidad y
FN, especificidad y FP (que es el indicador mas fácil de monitorizar), siempre teniendo en cuenta la distribución de edades de la población que se está cribando.

La sensibilidad plantea dificultades ya que precisa de un seguimiento postnatal de aquellas mujeres que fueron sometidas a screening, pero es un indicador necesario para justificar la aplicabilidad de métodos de cribado en población de bajo riesgo. El incremento observado en las últimas décadas de gestantes en edad superior a 35 años en países industrializados es paralelo al aumento de sensibilidad en los métodos de cribado en $2^{\circ}$ y $1^{\circ}$ trimestre por lo que, en opinión de los epidemiólogos, parece razonable mantener un nivel de corte que genere alrededor del 5\% de FP para la trisomía 21 y $1 \%$ para la trisomía 18. Para evitar aumentar costes de técnicas invasivas, así como las pérdidas fetales que conlleva, morbilidad y ansiedad materna y para que el análisis coste-efectividad sea favorable, la sensibilidad global para detección de SD debe mantenerse entre $60-70 \%$ para el $2^{\circ}$ trimestre y 80 $90 \%$ en el $1^{\circ}$ trimestre $^{1,30}$.

En la aplicación de las posibles alternativas propuestas deben considerarse las características de la población estudiada y los medios disponibles, como son la edad materna, actualmente con tendencia al aumento, la proporción de gestantes que consultan dentro del primer trimestre, la posibilidad de estudio ecográfico reproducible, la cualificación y recursos en la práctica de procedimientos invasivos y la disponibilidad de recursos de laboratorio. Estos factores sin duda pueden tener influencia en la decisión de aplicar una u otra estrategia.

Lo más complejo del screening con respecto a los posibles resultados adversos es la responsabilidad del médico para informar y discutir las alternativas, riesgos y beneficios, asesorando a cada paciente según sus preferencias. Algunas pacientes solicitarán preparación psicológica para el nacimiento de un niño con SD, otras optarán por IVE, mientras que otras no querrán someterse al scree ning mientras que la prueba diagnóstica vaya asociada a riesgo de pérdida fetal ${ }^{17}$.

Para facilitar la información que el médico de familia debe dar a las gestantes transcribimos en anexo el documento informativo elaborado por el grupo de consenso de la Sociedad Española de Ginecología y Obstetricia.

CORRESPONDENCIA:

$M^{a}$ Nélida Parga Soler

Centro de Salud "V Centenario"

C/ Real, 91

28700 San Sebastián de los Reyes. Madrid 


\section{Bibliografía}

1. Fortuni Estivill A, Borrell Vilaseca A, Cortés León M, Gallo Vallejo M, González de Agüero Laborda R, González Gónzalez A, et al. Screening de cromosomopatías fetales. En: Documentos de Consenso S.E.G.O 2000; 139-77.

2. Suárez Rodríguez MA, Ordoñez Bayón MJ. Diagnóstico prenatal. En: Brines Solanes J, Crespo Hernández M, Cruz Hernández M, Delgado Rubio A, Garagorri Otero JM, Hérnandez Rodriguez M, et al. Manual del Residente de Pediatria y sus Áreas Específicas. Guía formativa. Volumen I. Madrid: Asociación Española de Pediatria, 1997; 109-12.

3. Salamanca Ballesteros A, Padilla Vinuesa MC, LópezJurado R. Diagnóstico prenatal de las malformaciones congénitas. Una orientación para el Médico de Familia. En: Gallo Vallejo FJ, et al. Manual del Residente de Medicina de Familia y Comuitaria. $2^{a}$ ed. Barcelona: semFYC, 1997; 973-81.

4. U.S. Preventive Services Task Force. Guía de Medicina Clínica Preventiva. Edición Española. Barcelona: Medical Trends, 1998

5. Fernández Fernández I, Franch Nadal J, Martín Manzano JL, Navarro Pérez J, Orozco Beltrán D, Vázquez Garrido P. Programas Básicos de Salud. Programa del Adulto. Volumen 2. Diabetes Mellitus. Madrid: Doyma, 1998.

6. Arribas Mir L, Bailón Muñoz E, Galvez Ibañez M, Marzo Castillejo M, Melguizo Jiménez M, Navarro Martín JA, et al. Programas Básicos de Salud. Programa de la Mujer. Volumen 1. Manejo de los problemas de salud en el embarazo. Madrid: Doyma, 2000.

7. Martínez Fernández AR, Fuentes Corripio I, Rodríguez Ferrer M, Domingo Fernández CJ. Toxoplasmosis. Medicine $1998 ; 81: 3760-6$.

8. Centers for Disease Control and Prevention. Sensivity of the test for antibody to hepatitis B surface antigen. Unites States. MMWR 1993; 42: 707-10.

9. Grosheide PM, Wladimiroff JW, Heijtink RA, Mazel JA, Christiaens GCML, Nuijten ASM, et al. Proposal for routine antenatal screening at 14 weeks for hepatitis B surface antigen. BMJ 1995; 311 (4): 1197-9.

10. Larsen SA, Kraus SJ, Winttington WL. Diagnostic test. En: Larsen SA, Hunter EF, Kraus SJ, EDS. A manual of test for syphilis. Washington DC. American Public Health Association, 1990.

11. Zaaijer HL, Exel-Oehlrss PV, Kraaijeveld T, Altena E, Lelie PN. Early detection of antibodies to HIV-1 by third- generation assays. Lancet 1992; 340: 770-2.

12. Ades AE, Sculpher MJ, Gibb DM, Gupta R, Ratcliffe J. Cost effectiveness analysis of antenatal HIV screening in United Kingdom. BMJ 1999; 319 (7219): 1230-4.

13. Pareja Bezares A, Álvarez Pasquín MJ, Batalla Martínez C, Comín Bertrán E, Gómez Marco JJ, Niño Martín V, et al. Prevención de las enfermedades transmisibles. Atención Primaria 1999; 24 Suppl 1: 99-114.

14. Gilson GJ, Christensen F, Romero H, Bekes K, Silva L, Qualls CR. Prevention of group B streptococcus early-onset neonatal sepsis: comparision of the Center for Disease Control and prevention screening-based protocol to a risk-based protocol in infants at greater than 37 week's gestation. J
Perinatol 2000; 20 (8 Pt 1): 491-5.

15. Montero Alonso R, Barbadillo Izquierdo F, Anso Olivan S, Marrero Calvo M, Carpintero Martín I, Sastre Huerta E, et al. Neonatal sepsis caused by Streptococcus agalactiae. What should be done? An Esp Pediatr 1998; 48 (3): 288-92.

16. Group B stretococcal disease prevention practices of obstetrician-gynecologist. Obstet Gynecol 2001; 98 (1): 7-13.

17. Cate S. Maternal serum triple analyte screening in pregnancy. Am Fam Phys 2000; 62 (4): 738-40.

18. Taipale P, Hilesmaa V, Salonen R, Ylöstalo P. Increased nuchal translucency as a marker for fetal chromosomal defects. N Engl J Med 1997; 337: 1654-8.

19. Programa atención a la mujer. Atención Primaria Insalud. Area 9. Madrid 1999.

20. Vintzileos AM, Ananth CV, Fisher AJ, Smulian JC, DaySalvatore D, Beazoglou T, et al. An economic evaluation of second trimester genetic ultrasonography for prenatal detection of down syndrome. Am J Obstet Gynecol 1998; 179: 1214-9.

21. Kuppermann M, Goldberg JD, Nease RF, Washington AE. Who should be offered prenatal diagnosis? The 35-year old question. Am J Public Health. 1999; 89 (2): 160-3.

22. Wald NJ, Watt HC, Hackshaw AK. Integrated screening for Down's syndrome based on tests performed during the first and second trimesters. N Engl J Med 1999; 341: 461-7.

23. Bricker L, Crowley P, Neilson J, O'Dowd T. Antenatal care of low risk pregnancies: ultrasound. Clinical Evidence 2000; 4: 781-92 (BMJ publishing group)

24. Roberts T, Mugford M, Piercy J. Choosing options for ultrasound screening in pregnancy and comparision cost effectiveness: A decision analysis approach. Br J Obstet Gynaecol 1999; 106 (4): 397-8.

25. Cusick W, Vintzileos AM. Fetal Down syndrome screening: a cost effectiveness analysis of alternative screening programs. J Matern Fetal Med 1999; 8 (6): 243-8.

26. Vintzileos AM, Anauth CV, Smulian JC, Beazoglou T, Knupple RA. Routine second-trimester ultrasonography in United States: A cost-benefit analysis. Am J Obstet Gynecol 2000; 182 (3): 655-60.

27. Hayashi M, Kozu H, Takei H. Maternal urinary free $\beta$-subunit of human corionic gonadotrophin: creatinine ratios and fetal chromosomal abnormalities in the second trimester of pregnancy. Br J Obstet Gynaecol 1996; 103: 577-80.

28. Torgerson DJ. The impact of maternal age on the cost effectiveness of Down's syndrome screening. Br J Obstet Gynaecol 1996; 103 (6): 581-3.

29. Vintzileos AM, Anauth CV, Fisher AJ, Smulian JC, DaySalvatore D, Beazoglou T, et al. An economic evaluation of prenatal strategies for detection of trisomy 18 . Am J Obstet Gynecol 1998; 179 (5): 1220-4.

30. Bach Vallmajor C. Diagnóstico prenatal: cribado serológico de malformaciones congénitas. JANO 2000; 58 (1333): 857-64.

31. Ganniats TG. Justifying prenatal screening and genetic amniocentesis programs by cost effectiveness analyses: a re-evaluation. Med Decis Making 1996: 16 (1): 45-50. 
MÉTODOS DE CRIBADO DE CROMOSOMOPATÍAS. HOJA INFORMATIVA

Se encuentra usted al inicio de su embarazo. Como ya sabe, existen algunas alteraciones congénitas debidas a defectos cromosómicos, en las que falta o sobra de forma parcial o total alguno de los cromosomas de sus células. Una de las más frecuentes es el síndrome de Down, en el que existe un cromosoma en exceso (el número 21, por lo que se denomina también trisomía 21), que causa retraso mental y malformaciones en grado variable.

El síndrome de Down se presenta con mayor frecuencia en mujeres mayores de 35 años. Sin embargo, dos de cada tres nacidos con síndrome de Down lo hacen de madres menores de 35 años por la mayor frecuencia de embarazos en estas edades. Existen, además, otras alteraciones de los cromosomas que no están asociados a la edad materna.

Actualmente pueden realizarse pruebas no diagnósticas a partir del segundo y tercer mes del embarazo (10-14 semanas) cuyo resultado nos orientará a pensar si existe o no una cromosomopatía, es decir, una alteración en los cromosomas de su hijo que pueda tener graves consecuencias en su desarrollo físico y/ o mental. El objetivo final es conocer la integridad cromosómica de su hijo y, de no ser así, permitirle a usted acogerse a la Ley de la Interrupción Voluntaria del Embarazo.

Estas pruebas no suponen ningún riesgo y consisten en una extracción de sangre materna y una ecografía.

Los resultados de este análisis de sangre o de esta ecografía no son definitivos, sino puramente orientativos. Se ofrecen en forma de cifras, exponiéndosele a usted un índice de riesgo, un número, que puede ser mayor o menor al riesgo que, de forma natural se tiene por la edad materna, de tener un niño con anomalía cromosómica.

Se considera que es positiva la prueba cuando el riesgo estimado en dicho análisis es igual o superior a 1 en 270. Debe entenderse que el $5 \%$ de las pruebas con resultado "positivo" se dan en fetos cromosómicamente normales. Por ello este resultado no significa necesariamente que el feto esté afectado sino simplemente la posibilidad de que sea así. En ese caso se le informará de la posibilidad de confirmar el diagnóstico mediante una segunda prueba; ésta es invasiva y con cierto riesgo para el embarazo. Estas pruebas son la amniocentesis y la biopsia corial. La decisión de realizar o no esta confirmación diagnóstica seguirá siendo de usted. Estas pruebas se realizan de forma voluntaria y siempre podrá usted rehusarlas.

Hay aproximadamente un $0,5-1,5 \%$ de posibilidades de que el embarazo se interrumpa como consecuencia de la prueba definitiva (biopsia corial o amniocentesis) y de otros riegos asociados como son la inmunización Rh en caso de madres Rh negativas, la rotura o la infección de la bolsa amniótica, etc.

También hay aproximadamente un $30 \%$ de posibilidades de que, siendo la prueba negativa el feto sea cromosómicamente anormal. Es decir, la prueba puede resultar falsamente negativa en 1 de cada 3 casos de síndrome de Down.

Éstos son, a grandes rasgos, los datos más importantes que debe usted conocer. Consulte con su médico cualquier duda respecto a esta información. 